\title{
Outcome of Stone Fragmentation with Pneumatic and Ultrasonic Lithotripter During Percutaneous Nephrolithotomy
}

Shariful Islam Khan ${ }^{1}$, Md Nurul Hooda ${ }^{2}$, Md Safiul Alam Babul ${ }^{3}$, Mohammad Habibur Rahman ${ }^{4}$, Anup Roy Chowdhury ${ }^{5}$, Md Moynul Hoque Chowdhury ${ }^{6}$, Mohammad Zia Uddin ${ }^{7}$

Received: 05 - 04 - 2020

Accepted: 09 - 07 - 2020

Conflicts of interest: None

Keywords: Fragmentation, pneumatic, ultrasonic, lithotripter, percutaneous nephrolithotomy (PCNL)

\begin{abstract}
Background of the study: Percutaneous nephrolithotomy (PCNL) is the gold standard treatment for kidney stones larger than $2 \mathrm{~cm}$. PCNL has replaced open surgical removal of large or complex calculi at the most institutions. The success of PCNL is related to the ability to achieve an optimum access tract and proper fragmentation. A wide range of lithotripsy techniques are currently available. One of these is ultrasonic lithotripsy, in which the stones are fragmented and sucked out simultaneously. This technique induces minimal tissue injury and could be considered as a standard modality for PCNL. The pneumatic lithotripter uses pneumatic ballast, which crushes the stones without producing any thermal effects. Because this mechanical energy passes along the metal wire to the stone, the probe works like a chisel on the stone surface. This modality destroys all stones, regardless of their composition. There were very few studies had been done in this context in our country, so I had decided to do this study to compare the outcome of stone fragmentation with pneumatic and ultrasonic lithotripter during percutaneous nephrolithotomy.

Objective: To compare the outcome of stone fragmentation with pneumatic and ultrasonic lithotripter during percutaneous nephrolithotomy.

Materials \& Methods: This Prospective Interventional Study (Quasi Experimental Trial) was performed in Department of Urology, National Institute of Kidney Diseases and Urology, Sher-E-Bangla Nagar, Dhaka during the period from December 2014 to May 2016. A total of 60 subjects will be selected with renal calculi (as per inclusion E exclusion criteria), among these, half of the patients will be group A (using pneumatic lithotripsy) \& rest of the patients will be group B (using ultrasonic lithotripsy). The study subjects were underwent PCNL under general anesthesia, half of which used pneumatic lithotripter and rest used ultrasonic lithotripter for stone fragmentation. Above mentioned outcome variables were assessed both per-operative and post-operatively. Data were collected, processed $\mathcal{E}$ analyzed. Statistical analysis of relevant variables was done by unpaired Student's T test and Chi Square test. P value Â0.05 was considered significant.
\end{abstract}

Results: Total 60 patients were selected for study according to the selection criteria. Of the 60 subjects, 30 patients, those who were done PCNL by pneumatic lithotripsy were labeled as Group A and 30 patients, those who were done PCNL by ultrasonic lithotripsy, were labeled with Group B.

1. Medical Officer (Urology), NIKDU, Sher-E-Bangla Nagar, Dhaka-1207, Bangladesh,

2. Professor of Urology\& Director, NIKDU, Sher-E-Bangla Nagar, Dhaka-1207, Bangladesh

3. Assistant Professor cum Resident Surgeon, (Urology), NIKDU, Sher-E-Bangla Nagar, Dhaka-1207, Bangladesh

4. Assistant Registrar (Urology), NIKDU, Sher-E-Bangla Nagar, Dhaka-1207, Bangladesh

5. Assistant Registrar Transplant Surgery, NIKDU, Sher-E-Bangla Nagar, Dhaka-1207, Bangladesh

6. Assistant Professor (Urology), NIKDU, Sher-E-Bangla Nagar, Dhaka-1207, Bangladesh

7. Registrar (Urology), NIKDU, Sher-E-Bangla Nagar, Dhaka-1207, Bangladesh

Correspondence: Dr. Shariful Islam Khan, Medical Officer (Urology), NIKDU, Sher-E-Bangla Nagar, Dhaka-1207, Bangladesh. E-mail: drsharif35mmc@gmail.com 
Outcome of Stone Fragmentation with Pneumatic and Ultrasonic Lithotripter During Percutaneous Nephrolithotomy

Distribution of respondents in terms of different parameters is shown in tabulated form and statistical analysis was done in both groups to see statistical significance, $p$ value less than 0.05 was considered significant. The mean stone fragmentation time was $27.23 \pm 4.78$ (18-38) min in PCNL by pneumatic lithotripsy and those were $23.80 \pm 5.30$ (13-34) min in by ultrasonic lithotripsy which is statistically significant. Other variables of interest which includes stone clearance rate, post operative haematuria and post operative hospital stay, were not statistically significant.

Conclusion: Comparing the findings of the present study, results indicate that stone fragmentation time is lesser in ultrasonic lithotripsy than pneumatic lithotripsy in PCNL which decreases the overall operative time.

\section{Introduction}

Percutaneous nephrolithotomy (PCNL) is the gold standard treatment for kidney stones larger than $2 \mathrm{~cm}$ (Karakoc et al. 2015). PCNL has replaced open surgical removal of large or complex calculi at the most institutions (Matlaga and Assimos 2002). PCNL is a commonly used technique for the treatment of kidney stones with the advantages of lower morbidity rates, decrease in post-operative pain with faster postoperative recovery, minimum duration of hospital stay and formation of minimal scar tissue (Mohota et al. 2008).

The PCNL procedure can be divided into three steps, namely percutaneous access, tract dilatation and stone fragmentation. The success of PCNL is related to the ability to achieve an optimum access tract and proper fragmentation (Cho et al. 2010).

Percutaneous trocar nephrostomy for hydronephrosis opens the door of a new technique done by Goodwin and Co-workers (1955) about 50 years ago. Since then the procedure of percutaneous nephrostomy has been refined and has vastly enriched the armamentarium of the contemporary urologists (Streem et al. 1996). Initially, percutaneous nephrostomy was used only for urinary diversion; subsequently it has been used for more complex procedure such as stone extraction (Alam et al. 2010).

The revolution of minimally invasive surgery began in 1976 when Fernström and Johansson performed the first percutaneous nephrolithotomy (PCNL). Subsequent reports on PCNL from the Mayo Clinic (Segura et al, 1982), the University of Minnesota (Clayman et al. 1984), West Germany (Alken et al. 1981) and England (Wickham and Kellet 1981) established PCNL. Further refinement and advances in technique and equipments have allowed urologists to perform percutaneous stone removal with increasing efficacy and decreasing complications (Lingeman et al. 1995).
The pneumatic lithotripter uses pneumatic ballast, which crushes the stones without producing any thermal effects (Piergiovanni et al. 1994). Because this mechanical energy passes along the metal wire to the stone, the probe works like a chisel on the stone surface (Hofmann et al.1996). This modality destroys all stones, regardless of their composition. Another advantage of the pneumatic lithotripter is that its cost is low (Hofmann et al. 1994).

Ultrasonic lithotripter uses mechanical energy that is created by piezo-ceramic elements. The vibrations (23$27 \mathrm{~Hz}$ ) are transmitted through rigid probes, which results in a drilling action (Begun 1994 \& Fuchs 1988). This lithotripter allows stone fragments to be simultaneously aspirated through the hollow probe, which help to remove the stone particles. In particular, soft matrix stones such as phosphate-containing calculi can be readily sucked out. Ultrasonic lithotripsy is very safe because activating the probe when it is in contact with the urothelium results in superficial erosion. However, ultrasonic lithotripsy is somewhat less effective for very hard renal stone or hard stone with smooth surface (Hofmann 2002).

Due to consistent advancement in endoscopic technology and operative techniques in recent decades increased the success rate (more than 90\%) of PCNL and decreased the associated complications and morbidity (Marguet et al. 2005). Consequently, a wide range of lithotripsy techniques are currently available. One of these is ultrasonic lithotripsy, in which the stones are fragmented and sucked out simultaneously (Begun 1994). This technique induces minimal tissue injury and could be considered as a standard modality for PCNL. However, the fragmentation process can be laborious, especially if the stone is large or dense, and continuous irrigation is needed to prevent the probe from overheating. Notably to prevent failure of overheated devices, the ultrasonic lithotripter must be 
started at a power mode between 1 \& 2 (as recommended by the manufacturer), after which the power can be increased as need up to 3 (Lehman 2008).

Pneumatic lithotripsy may be more suitable for harder stones because it is more powerful than the other lithotripsy techniques and it can minimize tissue injury when applied cautiously. However, a disadvantage of this technique is that the stone fragments must be extracted with graspers, which is highly timeconsuming (Cho et al. 2010).

In the Department of Urology, Inje University College of Medicine, Seoul, Korea, C one Cho et al. (2010) showed total 74 patients treated with PCNL, 35 patients using pneumatic Lithotripter \& 39 patients ultrasonic lithotripter. Among them by using pneumatic lithotripter overall stone free rate was $85.7 \%$ \& by ultrasonic lithotripter overall stone free rate was 92.3\%. In the department of Urology, Haseki Teaching and Research Hospital, Istanbul, Turkey Muslumanoglu AY and his associates prospectively reviewed data from 275 patients treated with PCNL between 2002 and 2009. Among them patients using ultrasonic lithotripter stone free rate was $91.4 \%$ \& patients using pneumatic Lithotripter stone free rate was $85.1 \%$.

The aim of the study is to assess the outcome of stone fragmentation with pneumatic and ultrasonic lithotripter during PCNL.

\section{Materials and Methods}

This Prospective Interventional Study (Quasi Experimental Trial) was performed in Department of Urology, National Institute of Kidney Diseases and Urology, Sher-E- Bangla Nagar, Dhaka from December 2014 to May 2016 after taking the ethical clearance from the Ethical Committee of same institute. Sixty adult patients with renal stone admitted during the study period and underwent PCNL in Department of Urology, National Institute of Kidney Diseases and Urology, Sher-E- Bangla Nagar, Dhaka, were included in the study. Patients with bleeding disorder, positive urine culture, anatomical abnormality that hampers patient positioning, i.e scoliosis, were excluded from this study.

After proper counseling and detailed explanation of procedure written informed consent were taken from all patients before including them in the study. All patients were evaluated by detailed history, thorough physical examinations and relevant investigations. The investigations included full blood count, serum creatinine, plain $X$ ray $K U B$, abdominopelvic ultrasound, intravenous urograpy (IVU), hepatitis B and C screening, ECG, 2D echocardiogram, bleeding time \& clotting time, chest X-ray, urine analysis and culture. If urine culture showed any growth, sensitive antibiotics was administered and repeat urine culture was done following completion of antibiotic course and thereby negative urine culture was ensured before surgical intervention. Co-morbidities like hypertension, ischemic heart disease, diabetes mellitus, and bronchial asthma was addressed and controlled preoperatively. Anti-platelet drugs if used by the patient were stopped 7 days before surgery.

Patients were selected in every alternate sequence (odd numbers for pneumatic lithotripter, group A \& even numbers for ultrasonic lithotripter, group B). All patients underwent PCNL under general anesthesia and received intravenous broad spectrum third generation cephalosporin and amikacin as antibiotics.

Initially, a lithotomy position is used and a 5/6 F ureteric catheter placed transurethrally. Percutaneous access is created using an $18 \mathrm{G}$ access needle into the selected calyx under fluoroscopic or ultrasonic guidance keeping the patient in prone position. A straight-tipped guide wire is placed into the collecting system. The nephrostomy tract was dilated with fascial dilators and a 28/30 F Amplatz sheath positioned into the renal collecting system. The stone is disintegrated using pneumatic lithotripsy or ultrasonic lithotripsy as per group. Additional tracts were made as necessary with the aim of complete stone clearance. Nephroscopy with forceps is used to retrieve stones from calyx. Once complete clearance was confirmed fluoroscopically and endoscopically, a 5/6 F JJ stent was placed antegradely. On completion of the the procedure, the Amplatz sheath is removed after keeping a nephrostomy tube in situ.

On postoperative day 1 , nephrostomy tube was removed if the urine was not hemorrhagic and stone clearance was successful. The Foleys catheter was removed on $2^{\text {nd }}$ postoperative day. The JJ stent was removed after 6 weeks. A postoperative $\mathrm{Hb} \%$, serum creatinine, and urine culture were also obtained from each patient. The nephrostomy tube was left in place if a second PCNL session due to residual stones was planned. Re-PCNL, URS and ESWL were considered as accessory treatment alternatives when indicated. 
Outcome of Stone Fragmentation with Pneumatic and Ultrasonic Lithotripter During Percutaneous Nephrolithotomy

Outcome variables e.g. stone clearance, stone fragmentation time, post operative haematuria and post operative hospital stay were assessed by $\mathrm{Hb} \%$, serum creatinine, plain $\mathrm{x}$-ray KUB on $1^{\text {st }} \mathrm{POD}$ and urine $\mathrm{R} / \mathrm{M} / \mathrm{E} \& \mathrm{C} / \mathrm{S}$, serum creatinine, plain $\mathrm{x}$-ray KUB and USG of KUB at 1 month, 2 months and 3 months after the operation. Complications encountered during follow up were documented.

After meticulous checking and rechecking, data was compiled and statistical analysis - measures of dispersion (mean, standard deviation ) and the tests of significance (Unpaired Student's T test and $x^{2}$ test) were done using computer, based on statistical software (SPSS-statistical package for social science, Version- 21) and necessary help was taken from the resource personnel in the field of biostatistics. ' $p$ ' value $<0.05$ was considered as significant.

\section{Results}

Total 60 patients were selected for study according to the selection criteria. Of the 60 subjects, 30 patients, those who were done PCNL by pneumatic lithotripsy were labeled as Group A and 30 patients, those who were done PCNL by ultrasonic lithotripsy, were labeled with Group B.

\section{Age distribution between groups:}

The age of the patients in both groups of the present study ranged between 18 and 65 years and the majority between 18-40 years, of which 23 and 21 patients belong to group A and group $B$ respectively. Mean age \pm SD of Group A was $33.83 \pm 13.19$ (range 18- 65) and that of Group B was 36.27 \pm 12.20 (range 19-62) years. There was no significant difference of mean age between the two groups ( $p$ value $>0.05$ ).

\section{Sex distribution of the patients:}

Sex distribution was over all male $33(55 \%)$ and female 27 (45\%) with male female ratio 11:9. Karakoc et al. (2015) showed 83 patients over all male 53 (64\%) and female $33(36 \%)$ with male female ratio was 13:7. There was no significant difference of sex distribution between the two groups ( $p$ value $>0.05$ ).

Size of the stone was within 2-6 cm with varying consistency in both the groups. Mean size of the stones were $3.87 \pm 1.306 \mathrm{~cm}$ in group $A$ and the size of the stones were $3.83 \pm 1.289 \mathrm{~cm}$ in group B. Calculated $p$ value was 0.73 which is not significant ( $p>0.05)$.
Table-I: Comparison of stone size between groups

Stone size Group A(n=30) Group B (n=30) p

\begin{tabular}{lccccc}
$(\mathrm{cm})$ & No & $\%$ & No & $\%$ & value \\
\hline $2.0-3.0$ & 13 & $43.33 \%$ & 13 & $43.33 \%$ & \\
$3.1-4.0$ & 7 & $23.33 \%$ & 8 & $26.67 \%$ & 0.73 \\
$4.1-5.0$ & 8 & $26.67 \%$ & 7 & $23.33 \%$ & \\
$5.1-6.0$ & 2 & $6.67 \%$ & 2 & $6.67 \%$ & \\
Mean \pm SD & $3.46 \pm 1.06$ & $3.37 \pm 1.03$ & \\
\hline
\end{tabular}

Group A: patient with PCNL done by pneumatic lithotripsy. Group B: patient with PCNL done by ultrasonic lithotripsy Students T-test (Unpaired) done to analyze the data.

$\mathrm{SD}=$ standard deviation

An overall stone clearance rates was $86.7 \%$ (26) in group A and $13.33 \%$ (4) was not cleared. Among the group B an overall stone clearance rates was $93.3 \%$ (28) and $6.67 \%$ (2) was not cleared. Stone clearance rates were not significant $(\mathrm{p}>0.05)$.

Table II: Comparison of stone clearance between groups

\begin{tabular}{lccc}
\hline Group & Stone cleared & Stone not cleared & p value \\
Group A & $26(86.7 \%)$ & $4(13.33 \%)$ & 0.38 \\
$(n=30)$ & & & \\
$\begin{array}{l}\text { Group B } \\
(n=30)\end{array}$ & $28(93.3 \%)$ & $2(6.67 \%)$ & \\
\hline
\end{tabular}

Group A: patient with PCNL done by pneumatic lithotripsy. Group B: patient with PCNL done by ultrasonic lithotripsy Chi-square $\left(x^{2}\right)$ test done to analyze the data

The mean stone fragmentation time (min) of group A was $27.23 \pm 4.78$ and the mean stone fragmentation time(min) of group B was $23.80 \pm 5.30$ respectively. Stone fragmentation time was significant between the groups $(\mathrm{p}<0.05)$.

Table III: Comparison of stone fragmentation time between groups:

\begin{tabular}{lccccc}
\hline Stone & \multicolumn{2}{c}{ Group A } & \multicolumn{2}{c}{ Group B } & P \\
fragmentation & $(\mathrm{n}=30)$ & \multicolumn{2}{c}{$(\mathrm{n}=30)$} & value \\
time (min) & No & $\%$ & No & $\%$ & \\
$10-20$ & 2 & $6.67 \%$ & 9 & $30.00 \%$ & \\
$20-30$ & 20 & $66.66 \%$ & 17 & $56.67 \%$ & 0.01 \\
$30-40$ & 8 & $26.67 \%$ & 4 & $13.33 \%$ & \\
Mean \pm SD & $27.23 \pm 4.78$ & & $23.80 \pm 5.30$ & \\
\hline
\end{tabular}

Group A: patient with PCNL done by pneumatic lithotripsy. Group B: patient with PCNL done by ultrasonic lithotripsy Student's T-test (Unpaired) done to analyze the data. $\mathrm{SD}=$ standard deviation 
Mean haematuria (in days) occurred during postoperative period in group A was $2.73 \pm 0.74$ and in group B was $2.73 \pm 0.87$ Haematuria is almost same in duration in both the groups. The difference between the groups was statistically not significant $(p>0.05)$.

Table IV: Comparison of post-operative haematuria between groups

\begin{tabular}{|c|c|c|c|c|c|}
\hline \multirow[t]{2}{*}{$\begin{array}{l}\text { Haematuria } \\
\text { (days) }\end{array}$} & \multicolumn{2}{|c|}{$\begin{array}{c}\text { Group A } \\
(\mathrm{n}=30)\end{array}$} & \multicolumn{2}{|c|}{$\begin{array}{l}\text { Group B } \\
(\mathrm{n}=30)\end{array}$} & \multirow[t]{2}{*}{$\begin{array}{c}\mathrm{p} \\
\text { value }\end{array}$} \\
\hline & No & $\%$ & No & $\%$ & \\
\hline 2 & 13 & $43.34 \%$ & 14 & $46.66 \%$ & \\
\hline 3 & 13 & $43.34 \%$ & 12 & $40 \%$ & 0.87 \\
\hline 4 & 4 & $13.33 \%$ & 2 & $6.67 \%$ & \\
\hline 5 & 0 & 0 & 2 & $6.67 \%$ & \\
\hline Mean \pm SD & \multicolumn{2}{|c|}{$2.70 \pm 0.74$} & \multicolumn{2}{|c|}{$2.73 \pm 0.87$} & \\
\hline
\end{tabular}

Group A: patient with PCNL done by pneumatic lithotripsy. Group B: patient with PCNL done by ultrasonic lithotripsy. Students T-test (Unpaired) done to analyze the data. $\mathrm{SD}=$ standard deviation

The mean hospital stay in days was 2-5 days in both the groups. Days in hospital in post-operative period in group A and group B were 3.90 \pm 0.88 and 3.50 \pm 0.97 respectively. Days in hospital in post-operative period were not significant $(p>0.05)$.

Table V: Comparison of post-operative hospital stays between groups:

\begin{tabular}{|c|c|c|c|c|c|}
\hline \multirow[t]{2}{*}{$\begin{array}{l}\text { Hospital } \\
\text { stay(days) }\end{array}$} & \multicolumn{2}{|c|}{$\begin{array}{c}\text { Group A } \\
(n=30)\end{array}$} & \multicolumn{2}{|c|}{$\begin{array}{l}\text { Group B } \\
\qquad(\mathrm{n}=30)\end{array}$} & \multirow[t]{2}{*}{$\begin{array}{c}\mathrm{p} \\
\text { value }\end{array}$} \\
\hline & No & $\%$ & No & $\%$ & \\
\hline 2 & 2 & $6.67 \%$ & 5 & $16.67 \%$ & \\
\hline 3 & 7 & $23.33 \%$ & 10 & $33.33 \%$ & 0.10 \\
\hline 4 & 13 & $43.33 \%$ & 10 & $33.33 \%$ & \\
\hline 5 & 8 & $26.67 \%$ & 5 & $16.67 \%$ & \\
\hline Mean \pm SD & \multicolumn{2}{|c|}{$3.90 \pm 0.88$} & \multicolumn{2}{|c|}{$3.50 \pm 0.97$} & \\
\hline
\end{tabular}

Group A: patient with PCNL done by pneumatic lithotripsy. Group B: patient with PCNL done by ultrasonic lithotripsy Student's T-test (Unpaired) done to analyze the data. $\mathrm{SD}=$ standard deviation

\section{Discussion}

Renal stone treatment options have changed dramatically during the last two decades with the technological advancement of instruments (Resorlu et al. 2012). Previously, most patients requiring stone removal underwent open surgery. They need to make a large \& traumatic incision in the loin to extract a calculus from the renal collecting system has always been a major disadvantage of operations for renal stones. Today PCNL is the first choice treatment modality for the most renal stones larger than $2 \mathrm{~cm}$, multiple renal stones, and also for complex renal calculi (Akman et al. 2012).

The introduction and advances in various forms of intracorporeal lithotripters, especially ultrasonic and pneumatic devices, have improved the stone-free rates after PCNL, while concomitantly decreasing the risk of complications. These various intracorporeal lithotripters work on different physical principles of stone fragmentation (Teh, Zhong \& Preminger 1998). Pneumatic lithotripters work on the same principle as collision with a bullet; on impact, energy transmits compressed air pulses within a steel probe to the calculi to be fragmented (Atar et al. 2013). This technique offers safe, cheap, and effective clearance of calculi, and it is particularly useful for large and hard stones. Also, all stones can be destroyed regardless of their composition, but subsequent extraction of the stone fragments is required (Hofmann et al. 2002; Diri et al. 2012).

The revolution of minimally invasive surgery began in 1976 when Fernstrom \& Johannson first removed a renal calculus through a nephrostomy tract (Marianiet al. 2004). Subsequent reports on PCNL (percutaneous nephrolithotomy) from the Mayo Clinic (Segura et al. 1982) and the University of Minnesota (Clayman et al. 1984a) and from West Germany (Alken et al. 1981) and England (Wickham and Kellet. 1981) established PCNL, further refined and advances in technique and equipment have allowed urologists to perform percutaneous stone removal with increasing efficacy and decreasing complications (Lingeman et al. 1995b). Development in stone fragmentation, newer instruments \& improved fluoroscopy has increased the versatility of percutaneous surgery. Flexible, steerable nephroscopes have allowed access to all parts of the kidney. The indications for open surgery in stone diseases have thus become greatly reduced (Alam et al. 2010).

Present study has been conducted to compare the outcome of stone fragmentation with pneumatic and ultrasonic lithotripter during PCNL. This study included 60 patients of renal stone $\geq 2 \mathrm{~cm}$ in size as per selection criteria. 
The age of the patients in both groups of the present study ranged between 18 and 65 years and the majority between 18-40 years, of which 23 and 21 patients belong to group A and group $B$ respectively. Mean age \pm SD of Group A was 33.83 \pm 13.19 (range 18- 65) and that of Group B was 36.27 \pm 12.20 (range 19-62) years. There was no significant difference of mean age between the two groups ( $p$ value $>0.05$ ). The age range of present study is comparable with many other similar studies.

Sex distribution was over all male 33 (55\%) and female $27(45 \%)$ with male female ratio 11:9. Karakoc et al. (2015) showed 83 patients over all male 53 (64\%) and female 33 (36\%) with male female ratio was 13:7.

With varying consistency the mean stone size in group A was $3.46 \pm 1.06(2.0-6.0) \mathrm{cm}$ and that was in group B $3.37 \pm 1.03(2.0-6.0) \mathrm{cm}$. The stone sizes of both groups were compared and group A had larger stone but statistically not significant ( $\mathrm{P}$ value $>0.05$ ). Hofmann et al. (2002) in 68 patients with PCNL by pneumatic lithotripsy and ultrasonic lithotripsy, mean stone size of their study was $4.34 \pm 1.245(3-7) \mathrm{cm}$ in group A and that was $3.98 \pm 1.5(2-7) \mathrm{cm}$ in group $B$. A retrospective study by Kim et al. (2003) found that the mean stone size was $4.9(3-7) \mathrm{cm}$. Mean stone size was $6.7 \pm 3.6$ and $9.6 \pm 5.7 \mathrm{~cm}$ for pneumatic and ultrasonic lithotripsy respectively in a study by Muslumanglu et al. (2009). Stone size did not correlate significantly with both the groups.

In the present study, stone free rate was $86.7 \%$ in group A in which pneumatic lithotripsy was used and stone free rate was $93.3 \%$ in group B in which ultrasonic lithotripsy was used for PCNL respectively. The success rate of stone clearance in a study conducted by Cho et al. (2010) was $85.7 \%$ in pneumatic lithotripsy and $92.3 \%$ in ultrasonic lithotripsy respectively. Auge et al. (2002) in a retrospective study, found that overall stone free rate was $83.4 \%$ of patients in PCNL by using pneumatic lithotripsy and overall stone free rate was $87.6 \%$ of patient in PCNL by using ultrasonic lithotripsy respectively. Muslumanoglu et al. (2006) observed in their study that the success rate of stone clearance was $89.2 \%$ in pneumatic lithotripsy and $92.7 \%$ in ultrasonic lithotripsy respectively. So, present study is similar to that of previous studies.

In this study, mean stone fragmentation time was $27.23 \pm 4.78(18-38) \mathrm{min}$ in PCNL by pneumatic lithotripsy and those were $23.80 \pm 5.30$ (13-34) $\mathrm{min}$ in by ultrasonic lithotripsy. Mean operation time was
$101.03 \pm 15.021$ (55-135) $\mathrm{min}$ in PCNL by pneumatic lithotripsy and that was $88.60 \pm 16.456$ (48-125) $\mathrm{min}$ in by ultrasonic lithotripsy. Both the differencesare statistically significant (P value $<0.05)$. Lehman et al. (2008) showed mean stone fragmentation time was 37 min by pneumatic lithotripsy and that was $31.5 \mathrm{~min}$ by ultrasonic lithotripsy and mean operation time was $125 \pm 13.68 \mathrm{~min}$ for pneumatic lithotripsy and $110 \pm 12.34$ min for ultrasonic lithotripsy.Cho et al.(2010) in 74 patients found the mean operation time was $221 \pm 65$ min in pneumatic lithotripsy and that was $181 \pm 50.0$ min in ultrasonic lithotripsy for PCNL. Cheng et al. (2010) presented data where they found that mean operation time $139.1 \mathrm{~min}$ for pneumatic and $134.9 \mathrm{~min}$ for ultrasonic lithotripsy in PCNL. Xu et al. (2010) found that the mean operative time of ultrasonic lithotripsy 110 mins (90-180) and in pneumatic lithotripsy 123 mins (90-180).

In this study, Postoperative haematuria occurred up to 2 days in $13(43.34 \%)$ cases, up to 3 days 13 (43.34\%) and up to 4 days $4(13.33 \%)$ e.g. for $2-4$ days in group A patients and up to 2 days in $14(46.66 \%)$ cases, up to 3 days $12(40.0 \%)$, up to 4 days $2(6.67 \%)$ and up to 5 days $2(6.67 \%)$ e.g. $2-5$ days in group B patients. In this study, mean duration of haematuria in group A was $2.70 \pm 0.74$ days and in group B was $2.73 \pm 0.87$ days. Difference was not statistically significant among the groups. Cho et al. (2010) found that mean duration of haematuria in group A was $1.97 \pm 0.13$ days and in group B was 2.23 \pm 0.12 days. Diri et al (2012) reported that mean duration of haematuria was nearly same in both the groups. So, this present study is similar to that of previous studies.

In our study, it has been observed that mean length of post-operative hospital stay was $3.90 \pm 0.88$ (2-5) days in pneumatic lithotripsy and that was $3.50 \pm 0.97$ (2-5) days in ultrasonic lithotripsy respectively. The length of hospital stay in both the groups was nearly same, statistically not significant. Cho et al. (2010) found 74 patients that mean length of total hospital stay was $14.2 \pm 4.4$ days by pneumatic lithotripsy and that was $11.6 \pm 3.8$ days in ultrasonic lithotripsy respectively. Auge et al. (2002) reported that mean length of hospital stay was $3.67 \pm 0.21$ days and $3.42 \pm 0.22$ days in pneumatic and ultrasonic lithotripsy respectively. Muslumanglu et al. (2009) reported in their study the mean hospital stay was $2.4 \pm 1.1$ (1-7) days. In a retrospective study by Gregory et al. (2009) found that the hospitalization time was not different among the groups, with a mean stay of 3 days. 


\section{Conclusion}

Comparing the findings of the present study, results indicate that stone fragmentation time is lesser in ultrasonic lithotripsy than pneumatic lithotripsy in PCNL which decreases the overall operative time. However, other variables e.g. size of the stone, stone clearance, post operative haematuria and post operative hospital stay were not statistically significant.

\section{References}

1. Akman, T, Binbay, M, Ozgor,F, Ugurlu, M, Tekinarslan, E, Kezer, C, Aslan, R \& Muslumanoglu, AY 2011, 'Comparison of percutaneous nephrolithotomy and retrograde flexiblenephrolithotripsy for the management of 2 - 4 cm stones: a matched-pair analysis', BJU Int, vol. 109, pp. 1384-1389. doi:10.1111/j.1464410X.2011.10691.x

2. Akdeniz, E, Ýrkýlata, L, Demirel, HC, Saylýk, A, Bolat, MS, Pahinkaya, N, Zengin, M \&Atilla, MK 2015, 'A comparison of efficacies of holmium YAG laser, and pneumatic lithotripsy in the endoscopic treatment of ureteral stones', Turkish Journal of Urology, vol. 41, no. 2, pp. 73-7. DOI:10.5152/ tud.2014.46548.

3. Alam, AKMK, Siddique, MFH, Islam, MS, Amanullah, ATM, Haque, ME, Uddin, MM, Khan, SA, Islam, MF, Islam, AKMA \& Salam, MA 2010, 'Percutaneous Nephrolithotomy With Multiple Tracts: Comparison Of Morbidity With Single tract Procedure', Bangladesh Journal of Urology, vol-13, no. 1, pp. 21-33.

4. Auge, BK, Lallas, CD, Pietrow, PK, Zhong P, \& Preminger, GM 2002, 'Invitro comparison of standard ultrasound and pneumatic lithotrites with a new combination intracorporeal lithotripsy device', Urology, vol. 60, no. 1, pp. 28-32.

5. Cho, C, Yu, JH, Luck, Sung, LH, Chung, JY \& Noh, $\mathrm{CH}$ 2010, 'Comparison of percutaneous nephrolithotomy using pneumatic lithotripsy (litholast) alone or combination with ultrasonic lithotripsy', Korean J Urol, vol.51, pp. 783-787.

6. Diri, A, Resorlu, B, Asterci, M, Unsal, A \& Germiyonoglu, C 2012, 'Tissue effects of intracorporeal lithotripsy techniques during Percutaneous nephrolithotomy. Comparison of pneumatic and ultrasonic lithotripter', Urol Res, vol. 40 no. 4 , pp. $409-13$

7. Gregory, Lowe, Bodo, E, Knudsen 2009, 'Ultrasonic, pneumatic and combination intracorporeal lithotripsy for Percutaneous nephrolithotomy', J Endo Urol, vol. 23, pp. 166368.

8. Hofmann, R, Weber, J, Heidenreich, A, Varga, Z \&Olbert, P 2002, 'Experimental studies and first clinical experience with a new Lithoclast and ultrasound combination for lithotripsy', EurUrol, vol. 42, pp. 376-81.

9. Hossain, TMS, Asaduzzaman, M, Uddin, MN, Rahman, MH, Jahan, MU \&Bhuiyan, AKMZI 2013, ‘Percutaneous nephrolithotomy - a versatile technique for both simple and complex renal stone', Bangladesh Med Res Counc Bull, vol. 39, pp. 99-103.

10. Jou, YC, Shen, $\mathrm{CH}$, Cheng, MC, Lin, CT \& Chen PC 2007, 'High- power holmium: yttriumaluminiumgarnet laser for percutaneous treatment of large renal stones', Urology, vol.69, pp. 22-5.

11. Karakan, T, Diri, A, Hascicek, AM, Ozgur, BC, Ozcan, S \&Eroglu, M 2013, 'Comparison of Ultrasonic and Pneumatic Intracorporeal Lithotripsy Techniques during Percutaneous Nephrolithotomy', The Scientific World Journal, Volume 2013, Article ID 604361, 4 pages

12. Lehman, DS, Hruby, GW, Phillips, C, Venkatesh, R, Best, S \&Monga, M 2008, 'Prospective randomized comparison of a combined ultrasonic and pneumatic lithotrite with a standard ultrasonic lithotrite for percutaneous nephrolithotomy' J Endo Urol, vol. 22, pp. 285-9.

13. Mariani, AJ 2007, 'Combined electro hydraulic and holmium YAG laser uretero-scopicnephrolitrotipsy of large (greater than $4 \mathrm{~cm}$ ) renal calculi', Korean J Urol, vol. 177, pp. 168-73.

14. Zengin, K, Sener, NC, Bas, O, Nalbant, I \&Alisir, I 2014, 'Comparison of Pneumatic, Ultrasonic and Combination Lithotripters in Percutaneous Nephrolithotripsy', IntBraz J Urol, vol. 40, pp. 650-5. doi: 10.1590/S1677-5538. IBJU.2014.05.10. 